\title{
Treinamento físico sem prescrição não reduz níveis glicêmicos e medidas antropométricas de pacientes portadores de diabetes mellitus tipo 2 fisicamente
}

\section{ativos}

\author{
Exercise training without prescription does not decrease glycemic levels and anthropometric \\ measurements in individuals with diabetes mellitus type 2 \\ El ejercicio físico sin prescripción no es capaz de reducir los niveles glicémicos y las medidas \\ antropométricas de personas con diabetes mellitus tipo 2
}

Recebido: 16/09/2021 | Revisado: 26/09/2021 | Aceito: 29/09/2021 | Publicado: 02/10/2021

\author{
Ana Flávia Silveira \\ ORCID: https://orcid.org/0000-0003-3539-9579 \\ Universidade Federal de São Carlos, Brasil \\ E-mail: aninha.flaviasilveira@gmail.com \\ Alice Ramos da Silva \\ ORCID: https://orcid.org/0000-0001-9069-084X \\ Universidade Federal do Triângulo Mineiro, Brasil \\ E-mail: alice.ramos@hotmail.com \\ Ana Luísa Freitas Siqueira \\ ORCID: https://orcid.org/0000-0002-9887-4755 \\ Universidade Federal do Triângulo Mineiro, Brasil \\ E-mail: anasiqueira_@hotmail.com \\ Elisabete Aparecida Mantovani Rodrigues \\ ORCID: https://orcid.org/0000-0002-8404-1103 \\ Universidade Federal do Triângulo Mineiro, Brasil \\ E-mail: elisabeteamr@gmail.com \\ Joilson Meneguci \\ ORCID: https://orcid.org/0000-0003-2268-3589 \\ Universidade Federal do Triângulo Mineiro, Brasil \\ E-mail: joilson.meneguci@uftm.edu.br \\ Fernando Seiji da Silva \\ ORCID: https://orcid.org/0000-0002-6373-8817 \\ Universidade Federal do Triângulo Mineiro, Brasil \\ E-mail: fernando.silva@uftm.edu.br \\ Michelle Rodrigues Lagares Xavier \\ ORCID: https://orcid.org/0000-0002-8738-5550 \\ Universidade Federal do Triângulo Mineiro, Brasil \\ E-mail: michelle_lagares@hotmail.com \\ Eduardo Elias Vieira de Carvalho \\ ORCID: https://orcid.org/0000-0001-5026-335X \\ Universidade Federal do Triângulo Mineiro, Brasil \\ E-mail: eduardo.carvalho@uftm.edu.br \\ Luciana Duarte Novais Silva \\ ORCID: https://orcid.org/0000-0002-1200-5680 \\ Universidade Federal do Triângulo Mineiro, Brasil \\ E-mail: luciana.duarte.silva@uftm.edu.br
}

\begin{abstract}
Resumo
Objetivo: Avaliar os níveis glicêmicos, medidas antropométricas e qualidade de vida de pacientes com diabetes mellitus tipo 2 (DM2) que praticam atividade física regularmente, sem prescrição profissional. Métodos: Foram estudados 35 pacientes com DM2, sendo 16 homens, com idade de 60,1 \pm 9,5 anos, divididos em dois grupos, sendo eles: suficientemente ativos $(\mathrm{GSA}=24)$ e insuficientemente ativos $(\mathrm{GIA}=11)$, classificados pelas recomendações do American College Sports of Medicine, após aplicação do questionário de nível de atividade física IPAQ. Foram realizadas as avaliações de medidas antropométricas, glicemia de jejum, hemoglobina glicada, glicemia pós-prandial e qualidade de vida dos pacientes. Resultados: Foi documentado que $24(68,6 \%)$ dos voluntários realizavam $1.078,1 \pm 1.481,6 \mathrm{~min} / \mathrm{sem}$ e foram classificados como GSA e $11(31,4 \%)$ realizavam 58,6 $\pm 41,6 \mathrm{~min} / \mathrm{sem}$, sendo classificados como GIA ( $\mathrm{p}=0,0001)$. Não foi documentada diferença em nenhum dos índices glicêmicos avaliados entre os grupos. Na avaliação antropométrica apenas a circunferência do pescoço foi menor no GSA $(36,8 \pm 3,7 \mathrm{~cm})$ do
\end{abstract}


que do GIA $(40,2 \pm 6,1 \mathrm{~cm}), \mathrm{p}=0,04$. Por fim, na avaliação da qualidade de vida o domínio de preocupação social/vocacional foi menor no GSA $(8,3 \pm 1,8)$ do que no GIA $(9,5 \pm 1,7)$, p=0,04. Conclusão: A realização de atividade física sem prescrição adequada não melhora os índices glicêmicos, medidas antropométricas e qualidade de vida de pacientes com DM2. Significativa porcentagem dos pacientes com DM2 não realizam exercícios físicos regularmente, mesmo após indicação médica.

Palavras-chave: Diabetes mellitus tipo 2; Treinamento físico; Prescrição; Qualidade de vida.

\begin{abstract}
Objective: To assess blood glucose levels, anthropometric measurements and quality of life in patients with type 2 diabetes mellitus (DM2) who regularly practice physical activity without a professional prescription. Methods: 35 DM2 patients were studied, 16 men, aged $60.1 \pm 9.5$ years, divided into two groups, namely: sufficiently active $(\mathrm{GSA}=24)$ and insufficiently active $(\mathrm{GIA}=11)$, classified by the recommendations of the American College Sports of Medicine, after applying the IPAQ physical activity level questionnaire. Anthropometric measurements, fasting blood glucose, glycated hemoglobin, postprandial blood glucose and patients' quality of life were evaluated. Results: It was documented that $24(68.6 \%)$ of the volunteers performed 1,078.1 $\pm 1,481.6 \mathrm{~min} /$ week and were classified as GSA and $11(31.4 \%)$ performed $58.6 \pm 41.6 \mathrm{~min} /$ week, being classified as GIA ( $\mathrm{p}=0.0001)$. There was no documented difference in any of the glycemic indexes assessed between the groups. In the anthropometric evaluation, only the neck circumference was smaller in the GSA $(36.8 \pm 3.7 \mathrm{~cm})$ than in the GIA $(40.2 \pm 6.1 \mathrm{~cm}), \mathrm{p}=0.04$. Finally, in the assessment of quality of life, the domain of social/vocational concern was lower in the GSA $(8.3 \pm 1.8)$ than in the GIA (9.5 \pm 1.7$), \mathrm{p}=0.04$. Conclusion: The performance of physical activity without an adequate prescription does not improve glycemic indexes, anthropometric measures and quality of life of patients with DM2. A significant percentage of patients with DM2 do not exercise regularly, even after medical advice.
\end{abstract}

Keywords: Type 2 diabetes mellitus; Physical training; Prescription; Quality of life.

\title{
Resumen
}

Objetivo: Evaluar los niveles de glucosa en sangre, las medidas antropométricas y la calidad de vida en pacientes con diabetes mellitus tipo 2 (DM2) que practican regularmente actividad física sin prescripción profesional. Metodología: Se estudiaron 35 pacientes con DM2, 16 hombres, de 60,1 \pm 9,5 años, divididos en dos grupos, a saber: suficientemente activos (GSA = 24) e insuficientemente activos (GIA = 11), clasificados por las recomendaciones del American College Sports of Medicina, tras aplicar el cuestionario de nivel de actividad física IPAQ. Se evaluaron medidas antropométricas, glucemia en ayunas, hemoglobina glucosilada, glucemia posprandial y calidad de vida de los pacientes. Resultados: Se documentó que 24 (68,6\%) de los voluntarios realizaron 1.078,1 \pm 1.481,6 min/semana y fueron clasificados como GSA y $11(31,4 \%)$ realizaron 58,6 \pm 41,6 min / semana, siendo clasificados como GIA (p $=0,0001)$. No hubo diferencias documentadas en ninguno de los índices glucémicos evaluados entre los grupos. En la evaluación antropométrica, solo la circunferencia del cuello fue menor en la GSA $(36,8 \pm 3,7 \mathrm{~cm})$ que en la GIA (40,2 $\pm 6,1 \mathrm{~cm}), \mathrm{p}=0,04$. Finalmente, en la evaluación de la calidad de vida, el dominio de preocupación social / vocacional fue menor en el GSA $(8,3 \pm 1,8)$ que en el GIA $(9,5 \pm 1,7), p=0,04$. Conclusión: La realización de actividad física sin una prescripción adecuada no mejora los índices glucémicos, las medidas antropométricas y la calidad de vida de los pacientes con DM2. Un porcentaje significativo de pacientes con DM2 no hace ejercicio de forma regular, incluso después del consejo médico.

Palabras clave: Diabetes mellitus tipo 2; Entrenamiento físico; Prescripción; Calidad de Vida.

\section{Introdução}

A prática regular de atividade física vem sendo descrita como uma importante estratégia para promoção de saúde, prevenção e tratamento de diversas doenças crônicas (Warburton, Nicol \& Bredin, 2006). Estudos indicam que indivíduos que realizam treinamento físico regularmente, prescrito adequadamente, apresentam risco reduzido para morte prematura por todas as causas, incluindo as cardiovasculares, acidente vascular encefálico, hipertensão, diabetes mellitus, câncer, obesidade, depressão, bem como apresentam melhora na qualidade de vida e do sono (Warburton, Charlesworth, Ivey, Nettlefold \& Bredin, 2010; Piercy et al., 2018).

A fim de promover e manter a saúde, algumas recomendações (American College Sports of Medicine, 2007) foram estabelecidas. Com base em evidências científicas, indivíduos saudáveis com idade entre 18 e 65 anos devem praticar, no mínimo, 30 minutos de atividade física de intensidade moderada, cinco vezes por semana ou 20 minutos de atividade física de intensidade vigorosa, três vezes por semana, podendo ainda realizar uma combinação dessas intensidades. Além disso, atividades que mantêm ou aumentam a força e a resistência muscular também são recomendadas. Neste caso, devem-se 
realizar de 8 a 10 exercícios, pelo menos duas vezes por semana, utilizando os principais grupos musculares (Haskell et al., 2007).

Para mensurar o nível de atividade física alguns instrumentos são utilizados, dentre eles, o Questionário Internacional de Atividade Física (IPAQ) (Matsudo et al., 2001). O questionário IPAQ foi desenvolvido para avaliar o nível de atividade física em adultos, abrangendo quatro domínios: atividade física no lazer; atividades domésticas; atividade física relacionada ao trabalho e atividade física relacionada ao transporte, além da intensidade em que elas são realizadas (caminhada, moderada e vigorosa intensidade), mostrando ser um instrumento eficaz e confiável na mensuração do nível de atividade física desempenhado pelo indivíduo.

O treinamento físico aeróbio supervisionado, e prescrito adequadamente, por um profissional especializado é capaz de melhorar os níveis de glicose em jejum, o nível de insulina plasmática e a resistência à insulina (Shakil-Ur-Rehman, Karimi \& Gillani, 2017a; Gomes, Magalhães, Nascimento, Andrade, Silva \& Oliveira, 2020). Os níveis de colesterol Low Density Lipoprotein (LDL) podem ser reduzidos enquanto que os níveis do High Density Lipoprotein (HDL) aumentados (Shakil-UrRehman, Karimi \& Gillani, 2017b). Além disso, diminui a pressão arterial, os níveis de hemoglobina glicada (HbA1c), o Índice de Massa Corpórea (IMC), a circunferência abdominal, além de promover maior bem-estar aos pacientes (Hoogendoorn, Rutten, Hart, De Wolf \& Vos, 2018; Nicolucci et al., 2012; Machado, Baruqui Júnior, Bertolini, Resende, Silva \& Pereira, 2021).

No entanto, apesar dos estudos terem documentado que a atividade física regular nos níveis recomendados é capaz de promover e manter a saúde, não há um parâmetro adequado de doses de treinamento (intensidade, volume, frequência, entre outros parâmetros) quando se trata de um exercício sem prescrição prévia pelos profissionais da área, que o fazem de forma individualizada.

Diante disso, o objetivo do presente estudo é verificar se indivíduos portadores de diabetes mellitus tipo 2 (DM2) que se mantêm fisicamente ativos, segundo recomendações do American College Sports of Medicine, porém sem adequada prescrição do treinamento, apresentam benefícios nos níveis glicêmicos, medidas antropométricas e qualidade de vida.

\section{Métodos}

\subsection{Desenho do estudo}

Trata-se de um estudo clínico, de caráter transversal e descritivo (Estrela, 2018; Koche, 2011), que investigou a influência da prática de atividade física sem adequada prescrição por profissional habilitado, sobre as variáveis clínicas, antropométricas e de qualidade de vida de pacientes com DM2.

Inicialmente os pesquisadores triaram os pacientes em acompanhamento em um ambulatório de endocrinologia. Todos os pacientes selecionados para o estudo foram submetidos ao questionário IPAQ. Em seguida os pacientes foram classificados segundo seus níveis de atividade física, por meio das recomendações do American College Sports of Medicine. Posteriormente todos foram submetidos aos demais questionários bem como avaliações antropométricas. Por fim, os dados bioquímicos referentes ao nível de glicemia no sangue foram coletados dos prontuários dos pacientes.

O projeto foi aprovado pelo Comitê de Ética e Pesquisa da nossa instituição (parecer nº1984759) e após os pacientes serem informados em relação aos procedimentos e sigilo de informações referentes às suas identidades, todos assinaram o Termo de Consentimento em participar do estudo.

\subsection{Casuística}

Foram selecionados 38 pacientes, com o diagnóstico de DM2, que estavam em acompanhamento clínico no Setor de Endocrinologia do Hospital de Clínicas de nossa instituição. 
Foram considerados elegíveis para o estudo, pacientes com 18 anos ou mais, que apresentavam compensados do ponto de vista clínico, que fossem aderentes ao tratamento medicamentoso e que não possuíssem contra indicação para prática regular de atividade física não supervisionada. Não foram incluídos no estudo pacientes que realizavam treinamento físico adequadamente prescrito por profissionais de saúde. Foram excluídos do estudo dois pacientes que os dados dos níveis glicêmicos não estavam atualizados nos prontuários e um por não completar todas as fases dos protocolos propostos.

Desse modo, nosso grupo de investigação foi composto por 35 pacientes, com idade média de 60,1 $\pm 9,5$ anos, divididos em pacientes ativos e inativos. O grau de atividade física utilizado para classificação dos grupos seguiu as recomendações da American College of Sports Medicine (Haskell et al., 2007): Grupo Suficientemente Ativos (GSA) composto por 24 pacientes que realizavam treinamento físico por indicação médica, no entanto que não receberam qualquer orientação ou prescrição adequada sobre a intensidade, volume, frequência ou tipo de exercício a ser realizado; e Grupo Insuficientemente Ativos (GIA) - composto por 11 pacientes que não realizavam nenhum tipo de treinamento físico.

\subsection{Métodos de avaliação}

Para confirmação do diagnóstico clínico de diabetes, foram coletados nos prontuários eletrônico dos pacientes os dados de medida de HbA1c, glicemia em jejum e pós-prandial, além do tempo de diagnóstico de DM2 e uso de insulina. A versão curta do questionário de atividade física IPAQ foi aplicada por um pesquisador devidamente treinado e com experiência na utilização dessa ferramenta.

Foram mensurados o peso e altura dos pacientes, posteriormente todos foram submetidos as medidas de circunferência abdominal, panturrilha, braço e pescoço utilizando uma fita métrica (MacroLife de $150 \mathrm{~cm}$ ). Para padronização das medidas antropométricas foi definido: circunferência do pescoço foi realizada através de um ponto médio entre as vértebras cervicais $\mathrm{C} 1$ e $\mathrm{C}$, dos braços foram $10 \mathrm{~cm}$ acima da fossa cubital, do abdômen foram realizadas ao nível da cicatriz onfálica e das panturrilhas foram $10 \mathrm{~cm}$ abaixo da linha articular do joelho.

Em seguida foi aplicado o questionário de qualidade de vida DQOL Brasil (Correr et al., 2008), a pontuação do DQOL Brasil foi calculada segundo o seu score, ou seja, quanto mais alta a pontuação das respostas pior era a qualidade de vida do indivíduo. As respostas variavam de muito satisfeito, sendo sua pontuação igual a 1 a nada satisfeito, sendo sua pontuação igual a 5, no domínio satisfação. Nos demais domínios, impacto, preocupação social/vocacional e preocupação com o diabetes, as respostas variavam de nunca (pontuação 1) a sempre (pontuação 5).

Para a padronização do tempo gasto na atividade física foi utilizado o questionário IPAQ e para a padronização do nível de atividade física foi utilizado as recomendações do American College Sports of Medicine, sendo elas: 30 minutos de atividade física moderada 5 dias por semana ou 20 minutos de atividade física vigorosa 3 dias por semana. Pacientes que relataram, por meio do questionário IPAQ, atingirem esses níveis de atividade física foram considerados suficientemente ativos.

\subsection{Análise estatística}

As variáveis categóricas foram apresentadas como frequência e porcentagem. As variáveis contínuas foram apresentadas como média \pm desvio padrão da média.

O teste de normalidade de Kolmogorov-Smirnov foi utilizado para verificar a normalidade de distribuição das variáveis. Foi utilizado o teste de Mann Whitney para comparar as variáveis que não apresentaram uma distribuição normal (glicose em jejum, glicose pós-prandial, circunferência abdominal e qualidade de vida no domínio preocupação sociais/vocacionais) e o teste $\mathrm{T}$ Student não pareado para comparar aquelas variáveis com distribuição normal (HbA1c, circunferência de braço direito, braço esquerdo, panturrilha direita, panturrilha esquerda e pescoço, qualidade de vida nos 
seguintes domínios: satisfação, impacto, preocupação com diabetes e total). O teste de Fisher foi utilizado para analisar a heterogeneidade da frequência de distribuição dos dados (qualidade de vida e atividade física).

O nível de significância considerado foi de $5 \%(\mathrm{p}<0,05)$.

\section{Resultados}

As variáveis clínicas, antropométricas, qualidade de vida e capacidade física dos indivíduos portadores de DM2 estão apresentadas na Tabela 1 .

\subsection{Caracterização da amostra}

Dentre os 35 voluntários, $24(68,6 \%)$ foram classificados por meio do IPAQ como suficientemente ativos, pois realizavam um volume de treinamento igual ou superior ao recomentado pela American College Sports of Medicine, mesmo que sem prescrição por profissional habilitado, e $11(31,4 \%)$ como insuficientemente ativos, independente do sexo.

Não foram documentadas diferenças significantes entre os grupos estudados para as variáveis de idade, dados antropométricos e hemodinâmicos. Apenas a circunferência do pescoço foi significativamente menor no GSA quando comparados ao GSI $(\mathrm{p}=0,04)$, Tabela 1.

\subsection{Níveis glicêmicos}

Os valores de glicemia em jejum, HbA1c e glicemia pós-prandial apresentaram-se semelhantes entre os pacientes suficientemente ativos em comparação aos insuficientemente ativos ( $\mathrm{p}>0,05)$, Tabela 1 e Gráfico 1.

\subsection{Qualidade de vida}

Não houve diferença significativa na variável de qualidade de vida total ( $p>0,05)$. Apenas o domínio preocupação social/vocacional apresentou diferença entre os grupos estudados, sendo significativamente menor no GSA (p = 0,04), Tabela 1.

\subsection{Tempo de atividade física}

O tempo de atividade física realizado durante a semana pelos pacientes do GSA foi maior, apresentando diferença estatisticamente significativa quando comparados ao GSI ( $\mathrm{p}<0,0001$ ), Tabela 1 e Gráfico 2.

Tabela 1: Aspectos clínicos, antropométricos, qualidade de vida e capacidade física dos pacientes.

\begin{tabular}{lccc}
\hline \multicolumn{1}{c}{ VARIÁVEIS } & GSA $(\mathbf{n}=\mathbf{2 4})$ & GIA $(\mathbf{n}=\mathbf{1 1})$ & p \\
\hline Idade (anos) & $59,8 \pm 9,2$ & $60,7 \pm 10,6$ & 0,8 \\
Sexo & & & 0,06 \\
$\quad$ Masculino & 8 & 3 & 0,2 \\
$\quad$ Feminino & 16 & $81,7 \pm 15,9$ & 0,08 \\
Peso Corporal (kg) & $74,8 \pm 15,3$ & $166,8 \pm 7,6$ & 0,7 \\
Altura (m) & $160,9 \pm 9,6$ & $29,5 \pm 6,8$ & 0,7 \\
IMC (kg/m $\left.{ }^{2}\right)$ & $28,8 \pm 4,2$ & $133,3 \pm 18,9$ & 0,3 \\
PAS (mmHg) & $135,9 \pm 18,4$ & $77,5 \pm 12,3$ & 0,5 \\
PAD (mmHg) & $81,6 \pm 10,0$ & $73,5 \pm 8,6$ & \\
FC (bpm) & $76,5 \pm 12,5$ & \\
\hline
\end{tabular}


Tempo Diagnóstico (anos)

Glicemia em Jejum (mg/dL)

HbA1c (\%)

Glicemia Pós-prandial (mg/dL)

Uso de Insulina

Sim

Não

\section{Circunferência $(\mathbf{c m})$}

Braço Direito

Braço Esquerdo

Panturrilha Direita

Panturrilha Esquerda

Abdômen

Pescoço

\section{Qualidade de Vida}

Satisfação
Impacto
Preocupação Social/Vocacional
Preocupação Diabetes
Total

\section{Atividade Física}

Tempo de AF (min/sem)

$$
\begin{gathered}
12,7 \pm 8,1 \\
191,9 \pm 108,9 \\
9,1 \pm 2,2 \\
249,7 \pm 98,2
\end{gathered}
$$

20

4
$14,9 \pm 9,1$

0,5

$147,9 \pm 58,0$

0,2

$9,1 \pm 1,9$

0,9

$252,8 \pm 75,7$

0,9

10

1

0,5
0,2
0,9
0,9

\section{9}

$\begin{array}{cc}30,6 \pm 3,2 & 0,4 \\ 30,5 \pm 3,1 & 0,5 \\ 36,2 \pm 2,6 & 0,7 \\ 36,5 \pm 2,4 & 0,7 \\ 91,5 \pm 31,4 & 0,2 \\ 40,2 \pm 6,1 & \mathbf{0 , 0 4}\end{array}$

$$
\begin{gathered}
37,2 \pm 8,2 \\
37,7 \pm 8,9 \\
9,5 \pm 1,7 \\
8,1 \pm 3,5
\end{gathered}
$$$$
91,5 \pm 17,0
$$

kg: quilograma; m: metros; IMC: índice de massa corporal; $\mathrm{kg} / \mathrm{m}^{2}$ : quilograma por metro quadrado; PAS: pressão arterial sistólica; PAD: pressão arterial diastólica; mmHg: milímetros de mercúrio; FC: frequência cardíaca; bpm: batimentos por minuto; mg/dL: miligramas por decilitro; HbA1c: hemoglobina glicada; \%: porcentagem; cm: centímetros; AF: atividade física; min/sem: minutos por semana. Fonte: Autores

Gráfico 1 - Resultados obtidos de glicemia sanguínea entre os grupos GSA e GIA.

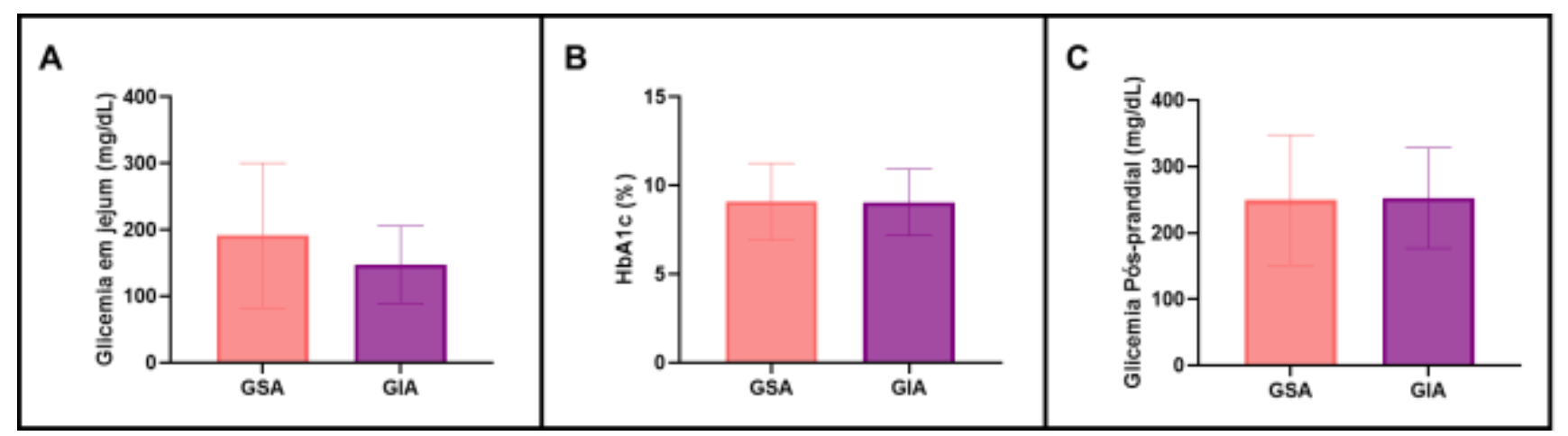

Representação gráfica da glicemia em jejum (A), HbA1c (B) e glicemia pós-prandial (C) nos grupos GSA e GIA, Mann Whitney Test/Test T Student. Fonte: Autores. 
Gráfico 2 - Tempo de atividade física realizada por semana entre os pacientes com DM2 suficientemente ativos e insuficientemente ativos.

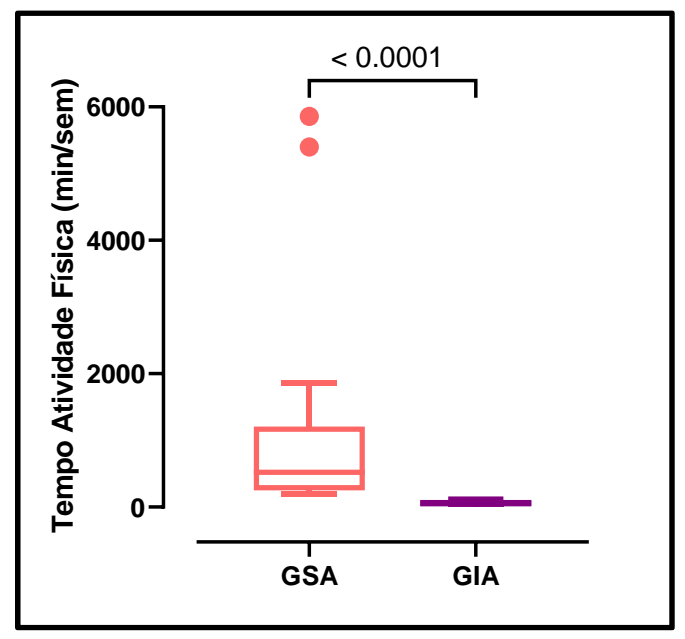

Representação gráfica do tempo de atividade física realizada por semanas nos grupos GSA e GIA, Mann Whitney Test. Fonte: Autores.

\section{Discussão}

Nossos principais achados evidenciaram que a prática de atividade física realizada de forma não prescrita e não programada, apenas por aconselhamento médico, não foi capaz de promover adaptações benéficas nas variáveis clínicas e antropométricas de pacientes portadores de DM2. Essas conclusões chamam a atenção para o fato de que embora esses pacientes sejam considerados fisicamente ativos, por atingirem a quantidade de atividade física atualmente recomendada pela American College Sports of Medicine (Haskell et al., 2007), o treinamento realizado sem a prescrição por um profissional especializado em fisiologia do exercício pode não ser suficiente para reduzir os níveis de glicemia sanguínea em jejum, pósprandial e hemoglobina glicada, bem como o peso corporal e circunferência abdominal de pacientes DM2.

É importante destacar também que dos 35 pacientes com DM2 estudados, $11(31,4 \%)$ pacientes não praticam atividade física, mesmo recebendo orientações quanto aos benefícios para sua saúde.

De acordo com a literatura, a atividade/exercício físico para ser benéfico, necessita de intensidade e volume adequados, planejados de forma individualizada e supervisionados por profissionais qualificados. Tanto os exercícios aeróbios quanto os resistidos são recomendados para indivíduos com DM2 por melhorarem o controle glicêmico, pressão arterial, risco cardiovascular, perda ou manutenção do peso e qualidade de vida (Colberg et al., 2010).

A fim de determinar os efeitos de um treinamento físico supervisionado por um especialista em exercícios, Balducci et al. (2010) acompanharam 563 pacientes sedentários com DM2, de ambos os sexos, por 12 meses. O treinamento padrão proposto para o grupo controle $(n=275)$ consistiu em aconselhamento individualizado, a cada três meses, para uma vida mais ativa através da prática de qualquer atividade que aumentasse o gasto energético basal e atingisse os níveis de atividade física recomendados. Já os demais pacientes $(n=288)$ participaram de um programa de treinamento aeróbio e resistido realizado duas vezes por semana, totalizando 150 minutos. Após comparar os dois grupos, verificou-se que o treinamento supervisionado produziu melhoras significativas na capacidade física (melhor aptidão física), variáveis clínicas (redução nos níveis de HbA1c, pressão arterial, colesterol e resistência à insulina) e antropométricas (diminuição da circunferência abdominal e IMC).

Em estudo semelhante, com o intuito de determinar os efeitos de um programa de treinamento de exercício aeróbio estruturado e supervisionado sobre os níveis glicêmicos, Shakil-Ur-Rehman, Karimi e Gillani (2017a) recrutaram 102 pacientes com diabetes tipo 2, de ambos os sexos e idade entre 40 a 70 anos. Enquanto o grupo experimental (n = 51) foi submetido a um programa de treinamento físico realizado em esteira em cinco fases, três vezes por semana, durante 25 
semanas, com acompanhamento médico de rotina e plano alimentar, o grupo controle $(\mathrm{n}=51)$ realizou apenas o acompanhamento médico e plano alimentar ao longo das 25 semanas. Ao final do período de intervenção, constatou-se que pacientes diabéticos treinados apresentaram diminuição dos níveis de glicemia em jejum, insulina plasmática, HbA1c e resistência à insulina, tanto na comparação pré e pós, quanto em relação aos pacientes diabéticos não treinados.

O treinamento físico realizado por caminhada regular, também beneficia indivíduos com sobrepeso, obesos e sedentários, podendo prevenir o desenvolvimento de DM2. Para tal, 50 indivíduos (60,6 \pm 10 anos), sendo 25 diabéticos e 25 com risco aumentado para DM2, foram convidados para integrar um programa de caminhada supervisionado por profissionais da saúde, entre eles, fisioterapeutas, nutricionistas, médicos e treinadores esportivos. A intervenção incluiu sessões iniciais de caminhadas de 6,8 ou 10 quilômetros, dependendo da aptidão física do participante, uma vez por semana, finalizando com 15 quilômetros caminhados por todos. Após 28 semanas de programa supervisionado, níveis menores no peso (88 vs $84 \mathrm{~kg}$ ), IMC (30,7 vs $\left.29,7 \mathrm{~kg} / \mathrm{m}^{2}\right)$ e circunferência abdominal (107,3 vs 103,9 cm) foram observados na população geral. Aqueles que apresentavam risco para o desenvolvimento de DM2 obtiveram ainda mais melhorias, como a redução da PAS (6,5 mmHg) e PAD (3,5 mmHg) (Hoogendoorn, Rutten, Hart, De Wolf \& Vos, 2018).

Embora a prática de atividade física não supervisionada não tenha promovido redução nas variáveis clínicas e na maioria das antropométricas em nosso estudo, o aconselhamento médico resultou em uma vida mais ativa, comprovado através do maior tempo de atividade física realizado semanalmente, o qual pode ter influenciado na diminuição da circunferência do pescoço e no domínio de preocupação social/vocacional da qualidade de vida. Resultados assim sugerem que o incentivo à prática de atividade física mesmo que de intensidade leve, pode ser o primeiro passo para que indivíduos ativos esporadicamente, com comorbidades ou não, adotem a prática regular de exercício físico, mantendo-a constante, e beneficiando a saúde em geral.

Sabe-se que a inatividade física/sedentarismo é um importante fator de risco para o agravamento de diversas doenças, como hipercolesterolemia, doença arterial coronariana, diabetes mellitus, acidente vascular encefálico e hipertensão arterial, bem como mortalidade. Quando comparados aos indivíduos fisicamente ativos que praticam 120 minutos de atividade física semanal de intensidade moderada, essa população de indivíduos sedentários/inativos apresenta risco aumentado que varia entre 20 a 30\% de mortalidade por todas as causas (Brownson, Boehmer \& Luke, 2005; Katzmarzyk \& Mason, 2009; Olshansky et al., 2005). Em contrapartida, níveis maiores de atividade física podem aumentar a longevidade (Moore et al., 2012), reduzir os custos e uso de terapia medicamentosa, melhorar a disposição para a execução de tarefas de vida diária, maximizando a qualidade de vida (Codogno, Fernandes \& Monteiro, 2012; Bertoldi, Hallal \& Barros, 2006).

Dados de estudo caso-controle realizados com pacientes adultos $(25-74$ anos) com DM2 $(n=150)$ e sem a doença $(n$ = 150) revelaram que pacientes diabéticos são mais inativos fisicamente quando comparados aos controles. De acordo com a pontuação do questionário IPAQ, enquanto pacientes sem diabetes realizaram níveis mais alto de atividades físicas que variaram de moderada a vigorosa intensidade, as atividades dos pacientes com a doença atingiram, em sua maioria, apenas os níveis de baixa e moderada intensidades, estando a quantidade de atividade física realizada inversamente relacionada com o avançar da idade (Ahmad et al., 2021).

Outro aspecto relevante sobre a saúde dos indivíduos diabéticos é que, além do nível de atividade física ser menor, esses pacientes também apresentam qualidade de vida inferior em comparação com os indivíduos não diabéticos (Kumar et al., 2016), como comprometimento da capacidade funcional, de socialização e saúde mental (Reis, Cunha, Bianchin, Freitas \& Castiglioni, 2019).

Sabe-se que a atividade física prescrita e realizada regularmente influencia positivamente no bem estar geral, melhorando a qualidade de vida (Rödjer, Jonsdottir \& Börjesson, 2016). Em estudo realizado com 95 pacientes com DM2 $(46,2 \pm 6,1$ anos), divididos entre grupo intervenção $(n=47)$ e controle $(n=48)$, foi demonstrado, através do questionário 
IPAQ, que após três meses de treinamento físico moderado prescrito, os pacientes apresentaram maior nível de atividade física além de melhora de aspectos físicos, psicológicos e ambientais da qualidade de vida quando comparados aos indivíduos controles (Soleimani Tapehsari, Alizadeh, Khamseh, Seifouri \& Nojomi, 2020).

Por fim, entendemos, a partir dos dados apresentados, que a prescrição adequada, por um profissional capacitado para a prática regular de exercícios físicos é mais importante do que a sua simples recomendação. Desse modo, destacamos que a prescrição do treinamento físico deve seguir o mesmo rigor metodológico que os médicos o fazem quando vão prescrever uma medicação a um paciente, orientando-o quanto a qual tipo de exercício realizar, qual dosagem/intensidade, volume, frequência e intervalos. Esses cuidados possibilitarão o paciente a realizar um treinamento mais seguro, personalizado e que induza uma maior quantidade de adaptações fisiológicas, trazendo benefícios para sua saúde em geral.

\section{Conclusão}

Pacientes com diabetes mellitus tipo 2 que praticam atividade física não prescrita e não programada, mesmo que considerados fisicamente ativos pela American College Sports of Medicine, apresentam níveis glicêmicos e medidas antropométricas semelhantes à de pacientes diabéticos fisicamente inativos. Grande parte dos pacientes diabéticos estudados não seguem as recomendações médicas de prática regular de atividade física. Ser fisicamente ativo, sem a prescrição correta do treinamento físico, não trouxe grandes benefícios para a qualidade de vida dos pacientes diabéticos.

Sugere-se para pesquisas futuras estudos com desenho longitudinal, com maior número de participantes, envolvendo um grupo de pacientes com DM2 submetidos a um treinamento físico programado e prescrito por um profissional habilitado.

\section{Referências}

Ahmad, I., Aung, M. N., Ueno, S., Khin, E. T., Latt, T. S., Moolphate, S., \& Yuasa, M. (2021). Physical Activity of Type 2 Diabetes Mellitus Patients and Non-Diabetes Participants in Yangon, Myanmar: A Case-Control Study Applying the International Physical Activity Questionnaires (IPAQ-S). Diabetes, Metabolic Syndrome and Obesity, 14, 1729-1739.

Balducci, S., Zanuso, S., Nicolucci, A., De Feo, P., Cavallo, S., Cardelli, P., Fallucca, S., Alessi, E., Fallucca, F., \& Pugliese, G. (2010). Effect of an intensive exercise intervention strategy on modifiable cardiovascular risk factors in subjects with type 2 diabetes mellitus: a randomized controlled trial: the Italian Diabetes and Exercise Study (IDES). Arch Intern Med, 170(20), 1794-1803.

Bertoldi, A. D., Hallal, P. C., \& Barros, A. J. (2006). Physical activity and medicine use: evidence from a population-based study. BMC Public Health, 6(224), $1-6$.

Brownson, R. C., Boehmer, T. K., \& Luke, D. A. (2005). Declining rates of physical activity in the United States: what are the contributors? Annu Rev Public Health, 26, 421-443.

Codogno, J. S., Fernandes, R. A., \& Monteiro, H. L. (2012). Prática de atividades físicas e custo do tratamento ambulatorial de diabéticos tipo 2 atendidos em unidade básica de saúde. Arquivos Brasileiros de Endocrinologia \& Metabologia, 56(1), 6-11.

Colberg, S. R., Sigal, R. J., Fernhall, B., Regensteiner, J. G., Blissmer, B. J., Rubin, R. R., Chasan-Taber, L., Albright, A. L., \& Braun, B. (2010). Exercise and type 2 diabetes: the American College of Sports Medicine and the American Diabetes Association: joint position statement. Diabetes Care, 33 (12), e147-167.

Correr, C. J., Pontarolo, R., Melchiors, A. C., Rossignoli, P., Fernández-Llimós, F., \& Radominski, R. B. (2008). Tradução para o português e validação do instrumento Diabetes Quality of Life Measure (DQOL-Brasil). Arquivos Brasileiros de Endocrinologia \& Metabologia, 52(3), 515-522.

Estrela, C. (2018). Metodologia Científica: Ciência, Ensino, Pesquisa. Editora Artes Médicas.

Gomes, J. G. F., Magalhães, C. F. C. B., Nascimento, Y. G. do, Andrade, W. T. B. de, Silva, A. H. B., \& Oliveira, G. A. L. de. (2020). Exercício físico e redução da resistência à insulina em indivíduos portadores de Diabetes Mellitus tipo 2: uma revisão bibliográfica. Research, Society and Development, 9(7), e463974375.

Haskell, W. L., Lee, I. M., Pate, R. R., Powell, K. E., Blair, S. N., Franklin, B. A., Macera, C. A., Heath, G. W., Thompson, P. D., \& Bauman, A. (2007). Physical activity and public health: updated recommendation for adults from the American College of Sports Medicine and the American Heart Association. Med Sci Sports Exerc, 39(8), 1423-1434.

Hoogendoorn, S. W., Rutten, G. E. H. M., Hart, H. E., De Wolf, C., \& Vos, R. C. (2018). A simple to implement and low-cost supervised walking programme in highly motivated individuals with or at risk for type 2 diabetes: An observational study with a pre-post design. Prev Med Rep, 7, 30-36.

Katzmarzyk, P. T., \& Mason, C. (2009). The physical activity transition. J Phys Act Health, 6(3), 269-280. 
Koche, J. C. (2011). Fundamentos de metodologia científica. Petrópolis: Vozes.

Kumar, P., Agarwal, N., Singh, C. M., Pandey, S., Ranjan, A., \& Kumar, D. (2016). Diabetes and quality of life - A pilot study. Int J Med Public Health, 5(6), 1143-1147.

Machado, P. G., Baruqui Júnior, A. M., Bertolini, N. O., Resende, N. M., Silva, G. C., \& Pereira, A. C. (2021). Moderate and high intensity exercise improves glycaemia, blood pressure and body composition in menopausal women with type 2 diabetes. Research, Society and Development, $10(8)$, e52810817571.

Matsudo, S., Araújo, T., Matsudo, V., Andrade, D., Andrade, E., Oliveira, L. C., \& Braggion, G. (2001). Questionário internacional de atividade física (IPAQ): estudo de validade e reprodutibilidade no brasil. Revista Brasileira De Atividade Física \& Saúde, 6(2), 5-18.

Moore, S. C., Patel, A. V., Matthews, C. E., Berrington de Gonzalez, A., Park, Y., Katki, H. A., Linet, M. S., Weiderpass, E., Visvanathan, K., Helzlsouer, K. J., Thun, M., Gapstur, S. M., Hartge, P., \& Lee, I. M. (2012). Leisure time physical activity of moderate to vigorous intensity and mortality: a large pooled cohort analysis. PLoS Med, 9(11), e1001335.

Nicolucci, A., Balducci, S., Cardelli, P., Cavallo, S., Fallucca, S., Bazuro, A., Simonelli, P., Iacobini, C., Zanuso, S., \& Pugliese, G. (2012). Relationship of exercise volume to improvements of quality of life with supervised exercise training in patients with type 2 diabetes in a randomised controlled trial: the Italian Diabetes and Exercise Study (IDES). Diabetologia, 55(3), 579-588.

Olshansky, S. J., Passaro, D. J., Hershow, R. C., Layden, J., Carnes, B. A., Brody, J., Hayflick, L., Butler, R. N., Allison, D. B., \& Ludwig, D. S. (2055). A potential decline in life expectancy in the United States in the 21st century. N Engl J Med, 352(11), 1138-1145.

Piercy K. L., Troiano, R. P., Ballard, R. M., Carlson, S. A., Fulton, J. E., Galuska, D. A., George, S. M., \& Olson, R. D. (2018). The Physical Activity Guidelines for Americans. JAMA, 320(19), 2020-2028.

Reis, A. C., Cunha, M. V., Bianchin, M. A., Freitas, M. T. R., \& Castiglioni, L. (2019). Comparison of quality of life and functionality in type 2 diabetics with and without insulin. Rev Assoc Med Bras, 65(12), 1464-1469.

Rödjer, L., H. Jonsdottir, I., \& Börjesson, M. (2016). Physical activity on prescription (PAP): self-reported physical activity and quality of life in a Swedish primary care population, 2-year follow-up. Scandinavian journal of primary health care, 34(4), 443-452.

Shakil-Ur-Rehman, S., Karimi, H., \& Gillani, S. A. (2017a). Effects of supervised structured aerobic exercise training program on fasting blood glucose level, plasma insulin level, glycemic control, and insulin resistance in type 2 diabetes mellitus. Pak J Med Sci, 33(3), 576-580.

Shakil-Ur-Rehman, S., Karimi, H., \& Gillani, S. A. (2017b) Effects of supervised structured aerobic exercise training program on high and low density lipoprotein in patients with type II diabetes mellitus. Pak J Med Sci, 33(1), 96-99.

Soleimani Tapehsari, B., Alizadeh, M., Khamseh, M. E., Seifouri, S., \& Nojomi, M. (2020). Physical Activity and Quality of Life in People with Type 2 Diabetes Mellitus: A Randomized Controlled Trial. International Journal of Preventive Medicine, 11(9), 1-6.

Warburton, D. E., Charlesworth, S., Ivey, A., Nettlefold, L., \& Bredin, S. S. (2010). A systematic review of the evidence for Canada's Physical Activity Guidelines for Adults. Int J Behav Nutr Phys Act, 7(39), 1-220.

Warburton, D. E., Nicol, C. W., \& Bredin, S. S. (2006). Health benefits of physical activity: the evidence. CMAJ, 174(6), 801-809. 